\title{
ON THE HODGE THEORY OF THE SYMMETRIC POWERS OF A CURVE
}

\author{
Sebastian Del Baño
}

Abstract

We describe the polarised Hodge structure on the symmetric powers of a smooth projective curve.

\section{Introduction}

Let $X$ be a topological space. The $n$-fold symmetric power of $X$ is the quotient of $X \times \cdots \times X$ by the natural action of the symmetric group $\mathfrak{S}_{n}$. We will denote it by $X^{(n)}$.

If $C$ is a smooth projective curve over the complex numbers $C^{(n)}$ is a compact complex manifold. If is of fundamental importance in the study of the jacobian variety of $C$ and other aspects of the geometry of $C$. In $[6]$ I. G. MacDonald computed the Betti numbers of the symmetric powers of a compact polyhedron. In the case of a smooth projective curve of genus $g, C$, the result is that the $i$-th Betti number is

$$
b_{i}=\sum_{\substack{a+b+c=n, b+2 c=i \\
a, b, c \in \mathbb{N} \cup\{0\}}}\left(\begin{array}{c}
b \\
2 g
\end{array}\right) .
$$

In [1] (see also [2]) we have extended this result to the theory of motives. We prove that the Chow motive of the variety $C^{(n)}$ is given by

$$
h\left(C^{(n)}\right) \simeq \bigoplus_{\substack{a+b+c=n \\ a, b, c \in \mathbb{N} \cup\{0\}}} \mathbb{1}^{\otimes a} \otimes \wedge^{b} h^{1}(C) \otimes \mathbb{L}^{\otimes c}
$$

(see loc. cit. for notations). In particular, we have an isomorphism of Hodge structures $H\left(C^{(n)}, \mathbb{Q}\right) \simeq \bigoplus_{a+b+c=n}^{a+c \in \mathbb{N} \cup\{0\}} \wedge^{b} H^{1}(C, \mathbb{Q})(-c)$.

2000 Mathematics Subject Classification. 14C25, 14D20.

Key words. Hodge theory, symmetric powers.

Partially supported by DGCYT grant PB93-0790. 
The purpose of this note is to extend this to an isomorphism of polarised Hodge structures. This can be of aid in computing the polarisation of the Hodge structure of the moduli space of stable vector bundles of rank two and fixed determinant of degree one. This would contain, in particular, the volume of the moduli space, found by Witten to be $\frac{1}{2 g-2} 2^{2 g-2}\left(2^{2 g-2}-2\right)\left|B_{2 g-2}\right|$ where $B_{i}$ is the $i$-th Bernoulli number.

\section{Preliminaries}

1.1. Polarization of symmetric powers. When a complex compact variety $X$ is given a polarization, i.e. an ample line bundle $\mathcal{L} \in \operatorname{Pic}(X)$, the symmetric power $X^{(n)}$ inherits in a natural way a polarization, $\mathcal{L}^{(n)}$. To see this consider the line bundle on $X^{n}$ defined by $\mathcal{L}^{n}=p_{1}^{*} \mathcal{L} \otimes \cdots \otimes$ $p_{n}^{*} \mathcal{L}$, where $p_{i}: X^{n} \longrightarrow X$ are the projections. The action of $\mathfrak{S}_{n}$ on $X^{n}$ lifts to $\mathcal{L}^{n}$ and the stabilizer $\mathfrak{S}_{n, x}$ of any point $x \in X^{n}$ acts trivially on the fibre $\mathcal{L}_{x}^{n}$. By Kempf's descent lemma ([4, Théorème 2.3]) the line bundle $\mathcal{L}^{n}$ is pullback via the quotient map $X^{n} \longrightarrow X^{n} / \mathfrak{S}_{n}=X^{(n)}$ of a line bundle $\mathcal{L}^{(n)}$. In order to see this line bundle is ample if $\mathcal{L}$ is so, proceed as follows: it is quite trivial to see that $\mathcal{L}^{n}$ is ample; ampleness of $\mathcal{L}^{(n)}$ is then a consequence of [5, Exercise III.5.7].

In the case of a smooth projective curve, $C$, any point gives an ample line bundle on $C$ and by the previous paragraph an ample line bundle on $C^{(n)}$. The cohomology class of this polarization is independent of the chosen point; it can be seen that it coincides with the class $\eta \in$ $H^{2}\left(C^{(n)}, \mathbb{Z}\right)$ defined in $[7]$ (see Subsection 1.3).

\subsection{Polarized Hodge structures.}

Definition $([\mathbf{3}, 2.1 .15])$. A polarized rational pure Hodge structure is a rational pure Hodge structure $M$ (say of weight $n$ ) together with a morphism of Hodge structures

$$
(\cdot, \cdot): M \otimes_{\mathbb{Q}} M \longrightarrow \mathbb{Q}(-n)
$$

such that on $M_{\mathbb{R}},(2 \pi i)^{n}(x, C y)$ defines a symmetric positive definite bilinear form where $C$ notes the Weil operator.

Examples. $\quad 1$. If $M$ and $N$ are polarized rational Hodge structures then so are $M \oplus N, M \otimes N$.

A polarization on a Hodge structure $M$ also induces a polarization on $\wedge^{i} M$, this is defined by $\left(v_{1} \wedge \cdots \wedge v_{i}, w_{1} \wedge \cdots \wedge w_{i}\right)=$ $\operatorname{det}\left(v_{k}, w_{l}\right)$.

2. The Tate Hodge structures $\mathbb{Q}(i)$ are trivially polarized. 
3. If $C$ is a smooth projective curve then $H^{1}(C, \mathbb{Q})$ is a polarized rational pure Hodge structure of weight 1 , the polarization is given by the cup product $H^{1}(C, \mathbb{Q}) \otimes_{\mathbb{Q}} H^{1}(C, \mathbb{Q}) \longrightarrow \mathbb{Q}(-1)$.

4. More generally if $X$ is a compact Kähler manifold with Kähler class $L \in H^{2}(X, \mathbb{Q})$ then the rational Hodge structure $H^{i}(X, \mathbb{Q})$ is naturally polarized. Assume $i \leq \operatorname{dim} X$ for simplicity, the Lefschetz decomposition states that $H^{i}(X, \mathbb{Q})=H_{0}^{i}(X, \mathbb{Q}) \oplus L H_{0}^{i-2}(X, \mathbb{Q}) \oplus$ $\cdots$, where $H_{0}^{*}(X, \mathbb{Q})$ is the primitive cohomology. The polarization is defined so that this is an orthogonal decomposition, if $\alpha, \beta \in$ $H_{0}^{i-2 s}(X, \mathbb{Q})$ then set $\left(L^{s} \alpha, L^{s} \beta\right)=(-1)^{\frac{i(i-1)}{2}+s} \int_{X} \alpha \wedge \beta \wedge L^{n-i+2 s}$.

1.3. The cohomology algebra of $C^{(n)}$. In this subsection we briefly recall the main results of $[7]$.

Let $C$ be a smooth projective algebraic curve over $\mathbb{C}$, let $\alpha_{1}, \ldots, \alpha_{2 g}$ be a basis of $H^{1}(C, \mathbb{Z})$ and $\beta$ a positive basis of $H^{2}(C, \mathbb{Z})$ such that $\alpha_{i} \alpha_{i+g}=\beta$ and $\alpha_{i} \alpha_{j}=0$ if $|i-j| \neq g$.

Then define $\xi_{i}=\left(\alpha_{i} \otimes 1 \otimes \cdots \otimes 1\right)+\cdots+\left(1 \otimes \cdots \otimes 1 \otimes \alpha_{i}\right)$ and $\eta=(\beta \otimes 1 \otimes \cdots \otimes 1)+\cdots+(1 \otimes \cdots \otimes 1 \otimes \beta)$. These are $\mathfrak{S}_{n}$-invariant elements in $H^{*}\left(C^{n}, \mathbb{Q}\right)$, therefore in $H^{*}\left(C^{(n)}, \mathbb{Q}\right)$. For $i \in\{1, \ldots, g\}$ define $\xi_{i}^{\prime}=\xi_{i+g}$ and $\alpha_{i}^{\prime}=\alpha_{i+g}$.

The cohomology algebra $H^{*}\left(C^{(n)}, \mathbb{Q}\right)$ is described by the following theorem.

Theorem $1([\mathbf{7}, 6.3])$. The cohomology algebra $H^{*}\left(C^{(n)}, \mathbb{Q}\right)$ is generated by $\xi_{1}, \ldots, \xi_{g}, \xi_{1}^{\prime}, \ldots, \xi_{g}^{\prime} \in H^{1}\left(C^{(n)}, \mathbb{Q}\right)$ and $\eta \in H^{2}\left(C^{(n)}, \mathbb{Q}\right)$ subject to the relations

$$
\xi_{i_{1}} \cdots \xi_{i_{a}} \cdot \xi_{j_{1}}^{\prime} \cdots \xi_{j_{b}}^{\prime} \cdot\left(\xi_{k_{1}} \xi_{k_{1}}^{\prime}-\eta\right) \cdots\left(\xi_{k_{c}} \xi_{k_{c}}^{\prime}-\eta\right) \cdot \eta^{q}=0
$$

whenever $\left\{i_{1}, \ldots, i_{a}\right\},\left\{j_{1}, \ldots, j_{b}\right\}$ and $\left\{k_{1}, \ldots, k_{c}\right\}$ are disjoint subsets of $\{1, \ldots, g\}$ and $a+b+2 c+q=n+1$.

As in [7] we shall use the following notation: for a subset $A$ of $\{1, \ldots, g\}$, if $A=\left\{i_{1}, \ldots, i_{a}\right\}$ with $i_{1}<\cdots<i_{a}$ we put $\xi_{A}=\prod_{k=1}^{a} \xi_{i_{k}}$, $\xi_{A}^{\prime}=\prod_{k=1}^{a} \xi_{i_{k}}^{\prime}$ and $\sigma_{A}=\prod_{k=1}^{a}\left(\xi_{i_{k}} \xi_{i_{k}}^{\prime}-\eta\right)$. We shall use upper case letters for subsets of $\{1, \ldots, g\}$ and the corresponding lowercase letters for the cardinality of the sets. Then (1) can be expressed in the following way: $\xi_{A} \xi_{B}^{\prime} \sigma_{C} \eta^{q}=0$ for any disjoint subsets of $\{1, \ldots, g\} A, B$ and $C$ with $a+b+2 c+q=n+1$.

We shall also need a basis of $H^{*}\left(C^{(n)}, \mathbb{Q}\right)$, this is given by the following result of MacDonald: 
Theorem 2 ([7, 3.2]). A basis of $H^{i}\left(C^{(n)}, \mathbb{Q}\right)$ is

(2) $\quad\left\{\xi_{i_{1}} \cdots \xi_{i_{p}} \eta^{q} \mid 0<i_{1}<\cdots<i_{p} \leq 2 g, \quad p+2 q=i\right\}$

with the further restriction $p \leq 2 n-i$ if $i>n$.

\section{The polarization on the cohomology of $C^{(n)}$}

Give $H^{*}\left(C^{(n)}, \mathbb{Q}\right)$ the polarization induced by the Kähler class $\eta$ and $\wedge^{i} H^{1}(C, \mathbb{Q})$ the polarization induced by that of $H^{1}(C, \mathbb{Q})$ then we have:

Theorem 3. There is a canonical isomorphism of polarized rational Hodge structures

$$
\varphi: H^{*}\left(C^{(n)}, \mathbb{Q}\right) \longrightarrow \bigoplus_{a+b+c=n} H^{0}(C, \mathbb{Q})^{\otimes a} \otimes \wedge^{b} H^{1}(C, \mathbb{Q}) \otimes H^{2}(C, \mathbb{Q})^{\otimes c} .
$$

Proof: We first claim that the primitive cohomology, $H_{0}^{i}(C, \mathbb{Q})$, has basis

$$
\left\{\begin{array}{c|c}
\xi_{A} \xi_{B}^{\prime} \sigma_{C} & A, B \text { and } C \text { disjoint subsets of }\{1, \ldots, g\} \\
a+b+2 c=i
\end{array}\right\} .
$$

To see this note first that these are indeed primitive classes for $\xi_{A} \xi_{B}^{\prime} \sigma_{C} \eta^{n-i+1}=0$ by (1) as $a+b+2 c+n-i+1=n+1$. To prove linear independence of (3) we write them in terms of the basis (2). If $C=\left(i_{1}, \ldots, i_{c}\right)$ then

$$
\begin{aligned}
\xi_{A} \xi_{B}^{\prime} \sigma_{C}= & \xi_{A} \xi_{B}^{\prime}\left(\xi_{i_{1}} \xi_{i_{1}}^{\prime}-\eta\right) \cdots\left(\xi_{i_{c}} \xi_{i_{c}}^{\prime}-\eta\right) \\
= & (-1)^{c} \xi_{A} \xi_{B}^{\prime} \eta^{c}+(-1)^{c-1} \xi_{A} \xi_{B}^{\prime}\left(\xi_{i_{1}} \xi_{i_{1}}^{\prime}+\cdots+\xi_{i_{c}} \xi_{i_{c}}^{\prime}\right) \eta^{c-1} \\
& +\cdots+\xi_{A} \xi_{B}^{\prime} \xi_{i_{1}} \xi_{i_{1}}^{\prime} \cdots \xi_{i_{c}} \xi_{i_{c}}^{\prime}
\end{aligned}
$$

Consider the vector space $V=H^{i}\left(C^{(n)}, \mathbb{Q}\right) / \eta H^{i-2}\left(C^{(n)}, \mathbb{Q}\right)$. It is clear from (2) that a basis for this quotient vector space is obtained by taking the classes of

$$
\left\{\xi_{j_{1}} \cdots \xi_{j_{i}} \mid 0<j_{1}<\cdots<j_{i} \leq 2 g\right\} .
$$

But by (4) the vectors (3) map to this basis of $V$. This proves the claim on the linear independence of (3) and it also proves that $\operatorname{dim} H_{0}^{i}\left(C^{(n)}, \mathbb{Q}\right)=\operatorname{dim} V$ coincides with the number of elements in $(3)$ hence it constitutes a basis for $H_{0}^{i}\left(C^{(n)}, \mathbb{Q}\right)$ as claimed.

Set $\alpha_{A}$ and $\alpha_{A}^{\prime}$ to stand for products of the $\left\{\alpha_{i}\right\}$ or $\left\{\alpha_{i}^{\prime}\right\}$ in the same manner as for $\xi_{A}$ and $\xi_{A}^{\prime}$. Define $\varphi$ on the primitive cohomology by

$$
\varphi\left(\xi_{A} \xi_{B}^{\prime} \sigma_{C}\right)=\alpha_{A} \wedge \alpha_{B}^{\prime} \wedge \alpha_{i_{1}} \wedge \alpha_{i_{1}}^{\prime} \wedge \cdots \wedge \alpha_{i_{c}} \wedge \alpha_{i_{c}}^{\prime}
$$

and extend it to all the cohomology by putting $\varphi\left(\xi_{A} \xi_{B}^{\prime} \sigma_{C} \eta^{q}\right)=$ $\varphi\left(\xi_{A} \xi_{B}^{\prime} \sigma_{C}\right) \otimes \beta^{q}$ where $\beta$ is the basis of $H^{2}(C, \mathbb{Q})$ defined above. Then $\varphi$ is an isomorphism of rational Hodge structures. 
We shall see that $\varphi$ preserves the polarization. It is enough to do so for primitive classes on which the polarization is given by

(5) $\left\langle\xi_{A} \xi_{B}^{\prime} \sigma_{C}, \xi_{\bar{A}} \xi_{\bar{B}}^{\prime} \sigma_{\bar{C}}\right\rangle=(-1)^{\frac{i(i-1)}{2}} \int_{C^{(n)}} \xi_{A} \xi_{B}^{\prime} \sigma_{C} \xi_{\bar{A}} \xi_{\bar{B}}^{\prime} \sigma_{\bar{C}} \eta^{n-i}$

In order to evaluate (5) first note that if $A \cap \bar{A} \neq \emptyset$ or $B \cap \bar{B} \neq \emptyset$ then clearly this product is zero, hence assume $A \cap \bar{A}=B \cap \bar{B}=\emptyset$.

If $A \cap \bar{C} \neq \emptyset, B \cap \bar{C} \neq \emptyset, \bar{A} \cap C \neq \emptyset$ or $\bar{B} \cap C \neq \emptyset$ the pairing (5) is zero: for example if $j \in A \cap \bar{C}$ then

$$
\xi_{A} \xi_{B}^{\prime} \sigma_{C}\left(\xi_{j} \xi_{j}^{\prime}-\eta\right) \eta^{n-i}=-\xi_{A} \xi_{B}^{\prime} \sigma_{C} \eta^{n-i+1}
$$

which is zero by (1) for $a+b+2 c+n-i+1=n+1$. Henceforth we shall assume $A \cap \bar{C}=B \cap \bar{C}=\bar{A} \cap C=\bar{B} \cap C=\emptyset$.

If $A \neq \bar{B}$ take $j \in \bar{B}-A$ (or if $\bar{B}-A=\emptyset$ take $j \in A-\bar{B}$ and proceed in the same manner), then $\xi_{A} \xi_{B}^{\prime} \xi_{j} \sigma_{C} \eta^{n-i}=0$ for $A, B \cup\{j\}$ and $C$ are disjoint and $a+b+1+2 c+n-i=n+1$. This way we see that if the pairing (5) is nonzero then $A=\bar{B}$ and $B=\bar{A}$ which we shall assume to be the case from now on.

Similarly if $C \neq \bar{C}$ the pairing is zero, for if we take $j \in \bar{C}-C$ then $\xi_{A} \xi_{B}^{\prime} \sigma_{C}\left(\xi_{j} \xi_{j}^{\prime}-\eta\right) \eta^{n-i}=0$ for $a+b+2 c+2+n-i=n+2$.

We conclude that the only nontrivial pairings are those of the form

$$
\begin{aligned}
\left\langle\xi_{A} \xi_{B}^{\prime} \sigma_{C}, \xi_{B} \xi_{A}^{\prime} \sigma_{C}\right\rangle & =\int_{C^{(n)}}(-1)^{\frac{i(i-1)}{2}} \xi_{A} \xi_{B}^{\prime} \xi_{B} \xi_{A}^{\prime}\left(\sigma_{C}\right)^{2} \eta^{n-i} \\
& =\int_{C^{(n)}}(-1)^{\frac{i(i-1)}{2}} \xi_{A} \xi_{A}^{\prime} \xi_{B}^{\prime} \xi_{B}\left(\sigma_{C}\right)^{2} \eta^{n-i} .
\end{aligned}
$$

Let $A=\left\{i_{1}, \ldots, i_{a}\right\}$ and $B=\left\{j_{1}, \ldots, j_{b}\right\}$. Note that

$$
\xi_{A} \xi_{A}^{\prime}=(-1)^{\frac{a(a-1)}{2}} \xi_{i_{1}} \xi_{i_{1}}^{\prime} \cdots \xi_{i_{a}} \xi_{i_{a}}^{\prime}
$$

Thus we have

$$
\begin{aligned}
& (-1)^{\frac{i(i-1)}{2}} \xi_{A} \xi_{A}^{\prime} \xi_{B}^{\prime} \xi_{B} \\
& \quad=(-1)^{\frac{i(i-1)+a(a-1)+b(b-1)}{2}+b} \xi_{i_{1}} \xi_{i_{1}}^{\prime} \cdots \xi_{i_{a}} \xi_{i_{a}}^{\prime} \xi_{j_{1}} \xi_{j_{1}}^{\prime} \cdots \xi_{j_{b}} \xi_{j_{b}}^{\prime}
\end{aligned}
$$


call this $S$. If $C=\left(k_{1}, \ldots, k_{c}\right)$ our pairing is the integral over $C^{(n)}$ of

$$
\begin{aligned}
S \sigma_{C}^{2} \eta^{n-i} & =S\left(\xi_{k_{1}} \xi_{k_{1}}^{\prime}-\eta\right)^{2} \cdots\left(\xi_{k_{c}} \xi_{k_{c}}^{\prime}-\eta\right)^{2} \eta^{n-i} \\
& =S\left(\eta-2 \xi_{k_{1}} \xi_{k_{1}}^{\prime}\right) \cdots\left(\eta-2 \xi_{k_{c}} \xi_{k_{c}}^{\prime}\right) \eta^{n-i+c} \\
& =\sum_{l=1}^{c} S(-2)^{l} \sigma_{l}\left(\xi_{k_{1}} \xi_{k_{1}}^{\prime}, \ldots, \xi_{k_{c}} \xi_{k_{c}}^{\prime}\right) \eta^{2 c-l+n-i}
\end{aligned}
$$

where $\sigma_{l}\left(\xi_{k_{1}} \xi_{k_{1}}^{\prime}, \ldots, \xi_{k_{c}} \xi_{k_{c}}^{\prime}\right)$ is the $l$-th symmetric function on the commuting variables $\xi_{k_{1}} \xi_{k_{1}}^{\prime}, \ldots, \xi_{k_{c}} \xi_{k_{c}}^{\prime}$.

By the next lemma

$$
\begin{aligned}
\left\langle\xi_{A} \xi_{B}^{\prime} \sigma_{C}, \xi_{B} \xi_{A}^{\prime} \sigma_{C}\right\rangle & =(-1)^{\frac{i(i-1)+a(a-1)+b(b-1)}{2}+b} \sum_{l=1}^{c}(-2)^{l}\left(\begin{array}{l}
j \\
c
\end{array}\right) \\
& =(-1)^{\frac{a^{2}+b^{2}+4 c^{2}+2 a b+4 a c+4 b c-a-b-2 c+a^{2}-a+b^{2}+b+2 c}{2}} \\
& =(-1)^{a b+b} .
\end{aligned}
$$

Now we turn to the evaluation of

(7) $\left\langle\varphi\left(\xi_{A} \xi_{B}^{\prime} \sigma_{C}\right), \varphi\left(\xi_{\bar{A}} \xi_{\bar{B}}^{\prime} \sigma_{\bar{C}}\right)\right\rangle$

$=\left\langle\alpha_{i_{1}} \cdots \alpha_{i_{a}} \alpha_{j_{1}}^{\prime} \cdots \alpha_{j_{b}}^{\prime} \alpha_{k_{1}} \alpha_{k_{1}}^{\prime} \cdots \alpha_{k_{c}} \alpha_{k_{c}}^{\prime}, \alpha_{\bar{i}_{1}} \cdots \alpha_{\bar{i}_{\bar{a}}} \alpha_{\bar{j}_{1}}^{\prime} \cdots \alpha_{\bar{j}_{\bar{b}}}^{\prime} \alpha_{\bar{k}_{1}} \alpha_{\bar{k}_{1}}^{\prime} \cdots \alpha_{\bar{k}_{\bar{c}}} \alpha_{\bar{k}_{\bar{c}}}^{\prime}\right\rangle$.

As before, it can be seen that the only nontrivial pairings are those where $A=\bar{B}, B=\bar{A}$ and $C=\bar{C}$, then (7) is the determinant of the matrix

$$
\left(\begin{array}{cccccc}
0 & -\operatorname{Id}_{b \times b} & & & \\
\operatorname{Id}_{a \times a} & 0 & & & & \\
& & 0-1 & & \\
& & & & \ddots & \\
& & & & 0-1 \\
& & & & & 1
\end{array}\right)
$$

where Id denotes the identity matrix and the symplectic matrix $\left(\begin{array}{ll}0 & -1 \\ 1 & 0\end{array}\right)$ is repeated $c$ times. It is easy to see that this determinant is $(-1)^{a b+b}$.

Lemma 4. If $0<i_{1}<\cdots<i_{a} \leq g$ then $\xi_{i_{1}} \xi_{i_{1}}^{\prime} \cdots \xi_{i_{a}} \xi_{i_{a}}^{\prime} \eta^{n-a}=\eta^{n}$. We also have $\int_{C^{(n)}} \eta^{n}=1$. 
Proof: The first assertion is easily proved by induction on $a$. It is trivial for $a=0$. By (1) $\left(\xi_{i_{1}} \xi_{i_{1}}^{\prime}-\eta\right) \xi_{i_{2}} \xi_{i_{2}}^{\prime} \cdots \xi_{i_{a}} \xi_{i_{a}}^{\prime} \eta^{n-a}=0$ therefore

$$
\xi_{i_{1}} \xi_{i_{1}}^{\prime} \cdots \xi_{i_{a}} \xi_{i_{a}}^{\prime} \eta^{n-a}=\xi_{i_{2}} \xi_{i_{2}}^{\prime} \cdots \xi_{i_{a}} \xi_{i_{a}}^{\prime} \eta^{n-a+1}
$$

which equals $\eta^{n}$ by the induction hypothesis.

For the second statement, note that the definition of $\eta$ (see Subsection 1.3) gives $\eta^{n}=n ! \cdot \beta \otimes n \otimes \beta$. Here, as in Subsection 1.3, we identify $H^{*}\left(C^{(n)}, \mathbb{Z}\right)$ with $\pi^{*} H^{*}\left(C^{(n)}, \mathbb{Z}\right) \subset H^{*}\left(C^{n}, \mathbb{Z}\right)$, so more precisely we would write $\pi^{*} \eta^{n}=n ! \cdot \beta \otimes n \otimes \beta$ (recall $\pi$ is the quotient $\left.\operatorname{map} C^{n} \longrightarrow C^{(n)}\right)$.

As $\pi$ is a finite morphism of degree $n ! \pi_{*} \pi^{*}$ is multiplication by $n !$, in particular $\eta^{n}=\pi_{*}(\beta \otimes \cdots \otimes \beta)$, therefore

$$
\int_{C^{(n)}} \eta^{n}=\int_{C^{n}} \beta \otimes \cdots \otimes \beta=\left(\int_{C} \beta\right)^{n}=1
$$

as claimed.

Our next result concerns the hard Lefschetz theorem. This is associated to a polarized variety $X$ of dimension $d$. If $L$ notes multiplication by the Chern class of the polarization, then the hard Lefschetz theorem states that

$$
L^{n-i}: H^{i}(X, \mathbb{Q}) \longrightarrow H^{2 d-i}(X, \mathbb{Q})
$$

is an isomorphism. The same statement for cohomology with coefficients in $\mathbb{Z}$ is false: take for example a hypersurface $X \subset \mathbb{P}^{d+1}$ of degree $n>$ 1 , then $\left(c_{1} \mathcal{O}_{X}(1)\right)^{d}=n$ and $L^{d}: H^{0}(X, \mathbb{Z}) \longrightarrow H^{2 d}(X, \mathbb{Z})$ is not an isomorphism. In contrast, for the varieties $C^{(n)}$ we have the following.

Corollary 5 (to proof of Theorem 3). The hard Lefschetz theorem for $C^{(n)}$ with its natural polarization $\eta$ holds with integer coefficients, that is

$$
\eta^{n-i} \wedge \cdot: H^{i}\left(C^{(n)}, \mathbb{Z}\right) \longrightarrow H^{2 n-i}\left(C^{(n)}, \mathbb{Z}\right)
$$

is an isomorphism.

Proof: Note, as remarked at the end of Subsection 1.1, that $\eta$ is the Chern class of a line bundle, hence $\eta$ is integral. To prove that $\xi_{1}, \ldots, \xi_{2 g}$ are also integral, let $p$ be a point on $C$ and consider the map

$$
\begin{aligned}
\iota: C & \longrightarrow C^{(n)} \\
x & \mapsto x+(n-1) p
\end{aligned}
$$


where we consider a point of $C^{(n)}$ as an effective divisor of degree $n$. By $[7,12.2] \iota$ induces an isomorphism

$$
\iota^{*}: H^{1}\left(C^{(n)}, \mathbb{Z}\right) \longrightarrow H^{1}(C, \mathbb{Z})
$$

and $\iota^{*} \xi_{i}=\alpha_{i}$, this shows that the classes $\xi_{i}$ are integral.

Let $i \leq n$ and let $V$ be the integral lattice generated by the following basis of $H^{i}\left(C^{(n)}, \mathbb{Q}\right)$

$$
\left\{\eta^{q} \xi_{A} \xi_{B}^{\prime} \sigma_{C} \mid 2 q+a+b+2 c=i\right\}
$$

(notation as in proof of Theorem 3). Also consider the lattice, $V^{\prime}$, generated by

$$
\left\{\eta^{n-i+q} \xi_{A} \xi_{B}^{\prime} \sigma_{C} \mid 2 q+a+b+2 c=i\right\} .
$$

By the previous $V, V^{\prime} \subset H^{*}\left(C^{(n)}, \mathbb{Z}\right)$.

Recall that Poincaré duality defines a unimodular pairing

$$
H^{i}\left(C^{(n)}, \mathbb{Z}\right) \otimes H^{2 n-i}\left(C^{(n)}, \mathbb{Z}\right) \longrightarrow \mathbb{Z} .
$$

In the proof of Theorem 3 we have seen that for each element in (8) there is a unique element of (9) such that the pairing (10) is nonzero and in this case it equals \pm 1 . This means that the pairing (10) restricted to $V$ and $V^{\prime}$ is still unimodular which readily implies that $V=H^{i}\left(C^{(n)}, \mathbb{Z}\right)$ and $V^{\prime}=H^{2 n-i}\left(C^{(n)}, \mathbb{Z}\right)$, in particular we get the statement in the corollary.

An interesting consequence of this corollary is that the isomorphism in Theorem 3, is in fact an isomorphism of polarized integral Hodge structures.

\section{References}

[1] S. DEL BaÑo, On the Chow motive of some moduli spaces, J. Reine angew. Math. 532 (2001), 105-132.

[2] S. DEL BAÑo, On the motive of moduli spaces of rank two vector bundles over a curve, Compositio Math. (2002) (to appear).

[3] P. Deligne, Théorie de Hodge. II, Inst. Hautes Études Sci. Publ. Math. 40 (1971), 5-57.

[4] J. N. Drezet and M. S. Narasimhan, Groupe de Picard des variétés de modules de fibrés semi-stables sur les courbes algébriques, Invent. Math. 97(1) (1989), 53-94.

[5] R. Hartshorne, "Algebraic geometry", Graduate Texts in Mathematics 52, Springer-Verlag, New York-Heidelberg, 1977.

[6] I. G. MacDonald, The Poincaré polynomial of a symmetric product, Proc. Cambridge Philos. Soc. 58 (1962), 563-568. 
[7] I. G. MacDonald, Symmetric products of an algebraic curve, Topology 1 (1962), 319-343.

Max-Planck-Institut für Mathematik

Gottfried-Claren-Straße 26

53225 Bonn

Germany

E-mail address: sebas@mpim-bonn.mpg.de

Current address:

NatWest Global Financial Markets

135 Bishopsgate

London EC2M 3UR

Great Britain

E-mail address: Sebastian.delBano@NatWestGFM.com

Primera versió rebuda el 17 de gener de 2001,

darrera versió rebuda el 15 de febrer de 2002. 